\title{
Poisson type relativistic perfect fluid spheres
}

\author{
Gonzalo García-Reyes* \\ Departamento de Física, Universidad Tecnológica de Pereira, A. A. 97, Pereira, Colombia
}

\begin{abstract}
Static spherically symmetric solutions of the Einstein's field equations in isotropic coordinates representing perfect fluid matter distributions from Newtonian potential-density pairs are investigated. The approach is illustrated with three simple examples based on the potential-density pairs corresponding to a harmonic oscillator (homogeneous sphere), the well-known Plummer model and a massive spherical dark matter halo model with a logarithmic potential. Moreover, the geodesic circular motion of test particles around such structures is studied. The stability of the orbits against radial perturbations is also analyzed using an extension of the Rayleigh criteria of stability of a fluid in rest in a gravitational field. The models considered satisfy all the energy conditions.
\end{abstract}

PACS numbers:

\section{INTRODUCTION}

Matter distributions with spherical symmetry have played an important role in astrophysics as models of dwarf spheroidal galaxies [1], bulges of disc galaxies [2, 3], galactic nuclei [4, [5], globular clusters [6], clusters of galaxies and dark matter haloes [7, 8]. In relativistic astrophysics, such matter configurations have been used as models of neutron stars, highly dense stars, gravastars, dark energy stars, quark stars, galactic nuclei and certain star clusters where relativistic effects are expected to be important. In relation to static spherically symmetric fields several exact solutions have been obtained over the years [9, 10]. In particular, anisotropic relativistic models of spherically symmetric matter distributions from various Newtonian potential-density pairs in isotropic coordinates were investigated in Refs. [11, 12] for Schwarzschild type space-times, also used in [13] for the same space-time, and in Ref. 14 for Majumdar-Papapetrou type fields. This same approach and spacetime was also used in 15] in the construction of three-dimensional anisotropic axisymmetric sources. On the other hand, perfect fluid matter distributions as a source of the gravitational field have also been widely studied in general relativity, mainly to describe models of stars, galaxies, and universes [16, 17].

In this work, we investigate the construction of perfect fluid sources for static spherically symmetric fields from a Newtonian potential-density pair using isotropic coordinates. These spacetimes contain two unknown metric functions. By substitution, one of the Einstein's field equations reduces to a nonlinear Poisson type equation. This equation is solved for one of the metric functions based on the assumption that in the Newtonian limit we should get a solution of the Poisson's equation of Newtonian gravity and based on a special form of the energy density. The other metric function is obtained solving the condition of pressure isotropy for a perfect fluid matter distribution. The method is illustrated with three simple examples based on the potential-density pairs of a homogeneous sphere, the well-known Plummer model and a logarithmic potential. These potential-density pairs have been used by some authors as models of dark matter haloes [1, 3, 34] and although such structures are essentially Newtonian, their relativistic description can be interesting not only from the merely theoretical point of view but also in the analysis of their interaction with large scale cosmic evolution and relativistic effects such as gravitational lenses or gravitational waves [18].

The paper is structured as follows. In Sect. II we present the method used for the construction of static spherically symmetric perfect fluid exact solutions from a given solution of Poisson's equation using isotropic coordinates. We also analyse the geodesic motion of test particles around the spherical distributions of matter and the stability of the orbits against radial perturbations using an extension of the Rayleigh criteria of stability of a fluid in rest in a gravitational field. In Sects. III-V the method is illustrated with three simple examples using as seed potential-density pairs those of a homogeneous sphere, the Plummer sphere and dark matter halo model with a logarithmic potential. Finally, in Sect. VI we summarize and discuss the results obtained.

*e-mail: ggarcia@utp.edu.co 


\section{RELATIVISTIC PERFECT FLUID SPHERES}

The line element for a static spherically symmetric spacetime in isotropic coordinates is given by [10]

$$
d s^{2}=e^{2 \nu} c^{2} d t^{2}-e^{2 \lambda}\left(d r^{2}+r^{2} d \Omega^{2}\right)
$$

where $d \Omega^{2}=d \theta^{2}+\sin ^{2} \theta d \varphi^{2}$, and $\nu$ and $\lambda$ are functions of only $r$. We use coordinates $\left(x^{0}, x^{1}, x^{2}, x^{3}\right)=(c t, r, \theta, \varphi)$. Einstein's gravitational field equations $G_{a b}=\left(8 \pi G / c^{4}\right) T_{a b}$ reduce to

$$
\begin{aligned}
& T_{t}^{t}=\frac{c^{2}}{4 \pi G} e^{-2 \lambda}\left[\nabla^{2} \lambda+\frac{1}{2} \nabla \lambda \cdot \nabla \lambda\right], \\
& T_{r}^{r}=\frac{c^{4}}{8 \pi G} e^{-2 \lambda}\left[\left(\lambda^{\prime}\right)^{2}+2 \lambda^{\prime} \nu^{\prime}+\frac{2}{r}\left(\lambda^{\prime}+\nu^{\prime}\right)\right], \\
& T_{\theta}^{\theta}=T_{\varphi}^{\varphi}=\frac{c^{4}}{8 \pi G} e^{-2 \lambda}\left[\lambda^{\prime \prime}+\nu^{\prime \prime}+\left(\nu^{\prime}\right)^{2}+\frac{1}{r}\left(\lambda^{\prime}+\nu^{\prime}\right)\right],
\end{aligned}
$$

where primes indicate differentiation with respect to $r$.

In terms of the orthonormal tetrad (comoving observer) $\mathrm{e}_{(a)}{ }^{b}=\left\{U^{b}, X^{b}, Y^{b}, Z^{b}\right\}$, where

$$
\begin{array}{rlrl}
U^{a} & =\frac{1}{\sqrt{g_{00}}} \delta_{0}^{a}=e^{-\nu} \delta_{0}^{a}, & X^{a}=\frac{1}{\sqrt{-g_{11}}} \delta_{1}^{a}=e^{-\lambda} \delta_{1}^{a}, \\
Y^{a}=\frac{1}{\sqrt{-g_{22}}} \delta_{2}^{a}=\frac{1}{r} e^{-\lambda} \delta_{2}^{a}, & Z^{a}=\frac{1}{\sqrt{-g_{33}}} \delta_{3}^{a}=\frac{1}{r \sin \theta} e^{-\lambda} \delta_{3}^{a} .
\end{array}
$$

the energy density is $\rho=T_{0}^{0} / c^{2}$ and the principal stresses $p_{i}=-T_{i}^{i} . U^{a}$ is the four-velocity of the matter distributions defined as the timelike vector of the orthonormal tetrad.

By making $e^{2 \lambda}=\left(1-\frac{\phi}{2 c^{2}}\right)^{4}$, we get for the energy density the following nonlinear Poisson type equation

$$
\nabla^{2} \phi=4 \pi G \rho\left(1-\frac{\phi}{2}\right)^{5}
$$

For a given physical energy density profile $\rho$, the metric function $\phi$ can be obtained by resolving this equation. A physically reasonable way to choose $\rho$ is by requiring that in the Newtonian limit it reduces to its Newtonian value $\rho_{N}$. A simple particular form of $\rho$ which satisfies such condition is

$$
\rho=\frac{\rho_{0}}{\left(1-\frac{\phi}{2}\right)^{5}} .
$$

Replacing this expression in (4) one finds in this case that the pair $\left(\phi, \rho_{0}\right)$ is a solution of the Poisson's equation. Hence $\left(\phi, \rho_{0}\right)=\left(\Phi, \rho_{N}\right)$ for any physical system. Therefore,

$$
\rho=\frac{\rho_{N}}{\left(1-\frac{\Phi}{2}\right)^{5}} .
$$

To obtain the other metric function $\nu$ an additional assumption must be imposed. For a perfect fluid source the momentum-energy tensor has the form [19 25]

$$
T^{a b}=\left(\rho c^{2}+p\right) U^{a} U^{b}-p g^{a b}
$$

and such assumption is the condition of pressure isotropy

$$
\lambda^{\prime \prime}+\nu^{\prime \prime}+\left(\nu^{\prime}\right)^{2}-\left(\lambda^{\prime}\right)^{2}-2 \lambda^{\prime} \nu^{\prime}-\frac{1}{r}\left(\lambda^{\prime}+\nu^{\prime}\right)=0
$$

which is a Riccati equation in either $\lambda^{\prime}$ or $\nu^{\prime}$. This condition is obtained by equating the field equations (2b) and (2c). It can also be cast as [26]

$$
L \mathcal{G}_{, x x}=2 \mathcal{G} L_{, x x}, \quad L \equiv e^{-\lambda}, \quad \mathcal{G} \equiv L e^{\nu}, \quad x \equiv r^{2}
$$


Thus, this approach allows to construct different static spherically symmetric solutions in isotropic coordinates representing perfect fluid matter distributions from a given solution of Poisson's equation. In addition, in order to have physically meaningful sources the parameters of the solutions must be chosen so that the energy conditions are satisfied. For a perfect fluid, the weak energy condition reads $\rho \geq 0$, whereas the dominant energy condition states that $|\rho| \geq|p|$. The strong energy condition requires that $\rho_{\text {eff }}=\rho+3 p \geq 0$, where $\rho_{\text {eff }}$ is the "effective Newtonian density".

An important quantity related to the circular motion of test particles around matter distributions is the circular speed (rotation profile) $v_{c}$. With respect to the comoving frame of reference (3a) - (3b), the 4-velocity of the particles $\mathbf{u}=d \mathbf{x} / d s$ has components

$$
u^{(a)}=e^{(a)}{ }_{b} u^{b},
$$

while the 3 -velocity

$$
v^{(i)}=c \frac{u^{(i)}}{u^{(0)}}=c \frac{e^{(i)}{ }_{a} u^{a}}{e^{(0)}{ }_{b} u^{b}} .
$$

For circular, equatorial orbits $\mathbf{u}=u^{0}(1,0,0, \omega / c)$, where $\omega=\frac{d \varphi}{d t}=c u^{3} / u^{0}$ is the angular speed of the test particles, and $v^{(\varphi)}$ is the only nonvanishing velocity component, and is given by

$$
\left[v^{(\varphi)}\right]^{2}=v_{c}^{2}=-\frac{g_{33}}{g_{00}} \omega^{2}
$$

This quantity represents the circular speed (rotation curves) of the particles measured by an inertial observer far from the source, and hence the equality. The angular speed $\omega$ can be calculated considering the radial motion of the particles along geodesics. The Lagrangian density for a massive test particle is defined as

$$
2 \mathcal{L}=g_{a b} \dot{x}^{a} \dot{x}^{b}=e^{2 \nu} c^{2} \dot{t}^{2}-e^{2 \lambda}\left(\dot{r}^{2}+r^{2} \dot{\theta}^{2}+r^{2} \sin ^{2} \theta \dot{\varphi}^{2}\right),
$$

where the overdot denotes derivative with respect to the affine parameter $s$ and Lagrange's equations read

$$
\frac{d}{d s}\left(\frac{\partial \mathcal{L}}{\partial \dot{x}^{a}}\right)-\frac{\partial \mathcal{L}}{\partial x^{a}}=0
$$

For circular orbits in the equatorial plane $\dot{r}=\dot{\theta}=0$, and the equation of motion for the radial coordinate gives

$$
\omega^{2}=-c^{2} \frac{g_{00, r}}{g_{33, r}}
$$

The tangential velocity is then

$$
v_{c}^{2}=c^{2} \frac{r \nu_{, r}}{1+r \lambda_{, r}}
$$

The stability of orbits against radial perturbations can be analyzed using an extension of the Rayleigh criteria for stability of a fluid at rest in a gravitational field [20, 27]. The stability condition is [28]

$$
\frac{d\left(h^{2}\right)}{d r}>0
$$

where $h$ is the specific angular momentum of a particle rotating at a radius $r$, defined as $h=p_{\varphi}=-c \frac{\partial \mathcal{L}}{\partial \dot{\varphi}}=-g_{33} u^{0} \omega$. $u^{0}$ is obtained normalizing $u^{a}$, that is requiring $g_{a b} u^{a} u^{b}=1$, so that

$$
\left(u^{0}\right)^{2}=\frac{1}{g_{00}+g_{33} \omega^{2} / c^{2}} .
$$

For the equatorial plane $\theta=\pi / 2$, we have

$$
h^{2}=\frac{e^{2 \lambda} r^{2} v_{c}^{2}}{1-\frac{v_{c}^{2}}{c^{2}}} .
$$




\section{HARMONIC OSCILLATOR TYPE SPHERES}

In Newtonian gravity the gravitational potential of a sphere of radius $a$ and constant mass density $\rho_{N}$ is

$$
\Phi= \begin{cases}-2 \pi G \rho_{N}\left(a^{2}-\frac{1}{3} r^{2}\right) & (r<a), \\ -\frac{4 \pi G \rho_{N} a^{3}}{3 r} & (r>a) .\end{cases}
$$

For $r<a$ the potential corresponds to a harmonic oscillator potential which has been used to model extended dark matter haloes with harmonic core [1]. The circular speed is

$$
v_{c N}=\sqrt{\frac{4 \pi G \rho_{N}}{3}} r .
$$

Solving the condition of pressure isotropy (9), the interior solution is

$$
\begin{aligned}
e^{\nu} & =\left[C_{1}\left(\frac{r^{2}}{a^{2}}-\frac{3(b+2)}{b}\right)^{4}+C_{2}\left(\frac{r^{2}}{a^{2}}-\frac{3(b+2)}{b}\right)^{-3}\right]\left[1+\frac{b}{2}\left(1-\frac{1}{3} \frac{r^{2}}{a^{2}}\right)\right]^{2}, \\
e^{\lambda} & =\left[1+\frac{b}{2}\left(1-\frac{1}{3} \frac{r^{2}}{a^{2}}\right)\right]^{2},
\end{aligned}
$$

where $b=2 \pi G \rho_{N} a^{2} / c^{2}$ is the parameter that measures the strength of the gravitational field, and $C_{1}$ and $C_{2}$ are constants of integration which obtain by demanding the continuity of the first and the second fundamental forms (Darmois conditions) at the boundary $r=a[29,30]$ between the above interior space-time and the exterior Schwarzschild metric which in isotropic coordinates reads

$$
\begin{aligned}
e^{\nu_{S c h}} & =\frac{1-\frac{a b}{3 r}}{1+\frac{a b}{3 r}} \\
e^{\lambda_{S c h}} & =\left(1+\frac{a b}{3 r}\right)^{2}
\end{aligned}
$$

Then it follows

$$
\begin{aligned}
C_{1} & =-\frac{9 b^{4}}{112(b+3)^{6}} \\
C_{2} & =\frac{432(b-4)}{7 b^{3}} .
\end{aligned}
$$

The main relativistic physical quantities associated with these matter distributions are

$$
\begin{aligned}
\rho & =\frac{\rho_{N}}{\left[1+\frac{b}{2}\left(1-\frac{1}{3} \tilde{r}^{2}\right)\right]^{5}}, \\
p & =-\frac{64 b c^{4}}{3 \pi G a^{2}} \frac{\left[\left[\left(1-\frac{1}{3} \tilde{r}^{2}\right) b+2\right]^{6}\left[\left(1-\frac{3}{2} \tilde{r}^{2}\right) b+2\right]+\frac{32}{729}(b-4)(b+3)^{6}\right]}{\left[\left[\left(-\frac{1}{3} \tilde{r}^{2}\right) b+2\right]^{7}+\frac{256}{729}(b-4)(b+3)^{6}\right]\left[\left(1-\frac{1}{3} \tilde{r}^{2}\right) b+2\right]^{5}}, \\
v_{c}^{2} & =4 b c^{2} \tilde{r}^{2} \frac{\left[-\left[\left(1-\frac{1}{3} \tilde{r}^{2}\right) b+2\right]^{7}+\frac{128}{2187}(b-4)(b+3)^{6}\right]}{\left.\left[\left(1-\frac{1}{3} \tilde{r}^{2}\right) b+2\right]^{7}+\frac{256}{729}(b-4)(b+3)^{6}\right]\left[\left(1-\frac{5}{3} \tilde{r}^{2}\right) b+2\right]}, \\
h^{2} & =\frac{a^{2} b c^{2}}{4} \tilde{r}^{4} \frac{\left[-\left[\left(1-\frac{1}{3} \tilde{r}^{2}\right) b+2\right]^{7}+\frac{128}{2187}(b-4)(b+3)^{6}\right]\left[\left(1-\frac{1}{3} \tilde{r}^{2}\right) b+2\right]^{4}}{\left[\left(1-\frac{1}{3} \tilde{r}^{2}\right) b+2\right]^{7}\left[\left(1+\frac{7}{3} \tilde{r}^{2}\right) b+2\right]+\frac{256}{729}(b-4)(b+3)^{6}\left[\left(1-\frac{7}{3} \tilde{r}^{2}\right) b+2\right]}
\end{aligned}
$$

where $\tilde{r}=r / a$. Since $r^{2} / a^{2} \leq 1$ the energy density is always positive in accordance with the weak energy condition. The other physical quantities will be analyzed using a graphical method. In figure 1 we plot the energy density $\tilde{\rho}=2 \pi G a^{2} \rho / c^{2}$, the isotropic pressure $\tilde{p}=\left(8 \pi G / c^{4}\right) p$, the circular speed $\tilde{v}_{c}^{2}=v_{c}^{2} / c^{2}$, the Newtonian rotation curves $\tilde{v}_{c N}^{2}=v_{c N}^{2} / c^{2}$ and the specific angular momentum $\tilde{h}^{2}=h^{2} /\left(c^{2} a^{2}\right)$ for the relativistic analogue of a homogeneous 
sphere with gravitational parameter $b=0.1,0.2,0.4$, as functions of $\tilde{r}$. We see that the energy density becomes constant as the gravitational field decreases, and the stresses are positive (pressure) and vanish at the boundary $r=a$. We also observer that the relativistic effects increase everywhere the speed of particles and they become more important as we move away from the central region. Moreover, we find that the increase in the gravitational field can make the orbits of particles unstable against radial perturbations. Indeed, the solution with parameter $b=0.4$ presents a region of instability of the orbits near the boundary. For these values of parameters the energy conditions are all satisfied.

\section{PLUMMER TYPE SPHERES}

A simple Newtonian potential-density pair is Plummer's model [6]

$$
\begin{aligned}
\Phi & =-\frac{G M}{\sqrt{r^{2}+a^{2}}}, \\
\rho_{N} & =\left(\frac{3 M}{4 \pi a^{3}}\right)\left(1+\frac{r^{2}}{a^{2}}\right)^{-\frac{5}{2}},
\end{aligned}
$$

where $a$ is a non-zero constant with the dimension of length. Plummer's sphere is used to model star clusters, the central spherical nucleus of spiral galaxies [31, 32] and also as models of dark matter haloes [3]. The circular speed is

$$
v_{c N}^{2}=\frac{G M r^{2}}{\left(r^{2}+a^{2}\right)^{3 / 2}} .
$$

The relativistic version of this model is a particular case of Buchdahl's solutions [33]. Therefore, the metric functions are

$$
\begin{aligned}
& e^{2 \nu}=\left(\frac{1-\frac{G M}{2 a c^{2} \sqrt{1+\frac{r^{2}}{a^{2}}}}}{1+\frac{G M}{2 a c^{2} \sqrt{1+\frac{r^{2}}{a^{2}}}}}\right)^{2}, \\
& e^{2 \lambda}=\left(1+\frac{G M}{2 a c^{2} \sqrt{1+\frac{r^{2}}{a^{2}}}}\right)^{4} .
\end{aligned}
$$

The main relativistic physical quantities associated with these structures are

$$
\begin{aligned}
\tilde{\rho} & =\frac{3 b}{2 \pi\left(\sqrt{1+\tilde{r}^{2}}+b\right)^{5}} \\
\tilde{p} & =\frac{b}{4 \pi\left(\sqrt{1+\tilde{r}^{2}}-b\right)\left(\sqrt{1+\tilde{r}^{2}}+b\right)^{5}}, \\
\tilde{v}_{c}^{2} & =\frac{2 b \tilde{r}^{2} \sqrt{1+\tilde{r}^{2}}}{\left(\sqrt{1+\tilde{r}^{2}}-b\right)\left[\left(1+\tilde{r}^{2}\right)^{3 / 2}+b\left(1-\tilde{r}^{2}\right)\right]} \\
\tilde{h}^{2} & =\frac{2 b \tilde{r}^{4}\left(\sqrt{1+\tilde{r}^{2}}+b\right)^{4}}{\left(1+\tilde{r}^{2}\right)^{3 / 2}\left[-4 b \tilde{r}^{2} \sqrt{1+\tilde{r}^{2}}+b^{2}\left(\tilde{r}^{2}-1\right)+\left(1+\tilde{r}^{2}\right)^{2}\right]}
\end{aligned}
$$

where $\tilde{\rho}=\frac{G a^{2}}{c^{2}} \rho, \tilde{p}=\frac{a^{3}}{c^{2} M} p, \tilde{v}_{c}^{2}=v_{c}^{2} / c^{2}, \tilde{h}^{2}=h^{2} / c^{2}, \tilde{r}=r / a$ and $b=G M /\left(2 a c^{2}\right)$. The energy density always is positive in agreement with the weak energy condition and for $b<1$ we have positive stresses, i.e. pressure.

In figure 2 we graph, as functions of $\tilde{r}$, the relativistic and Newtonian density profiles $\tilde{\rho}$ and $\tilde{\rho}_{N}=\frac{G a^{2}}{c^{2}} \rho_{N}$, and the isotropic pressure $\tilde{p}$ for Plummer type spheres with gravitational parameter $b=0.2,0.4,0.52$. We find that the energy density presents a maximum at $r=0$ and then decreases rapidly with $r$. We also find that when the gravitational 
field is increased the relativistic density profile always decreases at the central region of the matter distribution but then increases. In turn, the relativistic corrections decrease everywhere the energy density.

In figure 3 we plot the relativistic and Newtonian rotation curves $\tilde{v}_{c}^{2}$ and $\tilde{v}_{c N}^{2}=v_{c N}^{2} / c^{2}$, and the specific angular momentum $\tilde{h}^{2}$, also as functions of $\tilde{r}$. We see that both the gravitational field and relativistic effects increase the circular speed of particles and also that such corrections are more important in the regions around its maximum value and, as expected, for velocities comparable to the speed of light. We also find that the increase in the gravitational field can make the orbits of particles unstable against radial perturbations. Thus, the solution with parameter $b=0.52$ presents a region of instability of the orbits. For this value of the parameter the dominant energy condition is also satisfied.

\section{LOGARITHMIC POTENTIAL TYPE SPHERES}

Dark matter haloes can be modeled in Newtonian theory with a logarithmic potential of the form [34]

$$
\Phi=\frac{1}{2} v_{0}^{2} \ln \left(\frac{r^{2}+a^{2}}{b}\right)
$$

where $a$ and $b$ are constants, and $v_{0}$ is circular speed at large radii, also a constant. When $a=0$ this potential is often referred to as the singular isothermal sphere. The mass density distribution is

$$
\rho_{N}=\frac{v_{0}^{2}\left(r^{2}+3 a^{2}\right)}{4 \pi G\left(r^{2}+a^{2}\right)^{2}}
$$

and the circular speed at radius $r$ is

$$
v_{c N}=\frac{v_{0} r}{\sqrt{r^{2}+a^{2}}}
$$

This potential yields an asymptotically flat rotation curve.

Solving (9) a particular solution is

$$
\begin{aligned}
& e^{\nu}=C\left(r^{2}+a^{2}\right)\left[1-\frac{1}{4} \frac{v_{0}^{2}}{c^{2}} \ln \left(\frac{r^{2}+a^{2}}{b}\right)\right]^{6}, \\
& e^{\lambda}=\left[1-\frac{1}{4} \frac{v_{0}^{2}}{c^{2}} \ln \left(\frac{r^{2}+a^{2}}{b}\right)\right]^{2},
\end{aligned}
$$

where $C$ is an integration constant. The general solution is complicated and is not presented since it introduces unphysical properties to the solution. The main relativistic physical quantities associated with the distribution of matter are

$$
\begin{aligned}
\tilde{\rho} & =\frac{\tilde{v}_{0}^{2}\left(r^{2}+3 a^{2}\right)}{\left(r^{2}+a^{2}\right)^{2}}\left[1-\frac{1}{4} \tilde{v}_{0}^{2} \ln \left(\frac{r^{2}+a^{2}}{b}\right)\right]^{-5} \\
\tilde{p} & =\frac{2048 \tilde{v}_{0}^{2}\left(\left(7 \tilde{v}_{0}^{2}-4\right) r^{2}+\left(5 \tilde{v}_{0}^{2}-4\right) a^{2}\right) \ln \left(\frac{r^{2}+a^{2}}{b}\right)+\left(28672 \tilde{v}_{0}^{4}-49152 \tilde{v}_{0}^{2}+16384\right) r^{2}-32768 a^{2}\left(\tilde{v}_{0}^{2}-\frac{1}{2}\right)}{4096\left(r^{2}+a^{2}\right)^{2}\left[1-\frac{1}{4} \tilde{v}_{0}^{2} \ln \left(\frac{r^{2}+a^{2}}{b}\right)\right]^{6}} \\
\tilde{v}_{c}^{2} & =\frac{2 r^{2}\left[\tilde{v}_{0}^{2} \ln \left(\frac{r^{2}+a^{2}}{b}\right)+6 \tilde{v}_{0}^{2}-4\right]}{\tilde{v}_{0}^{2}\left(r^{2}+a^{2}\right) \ln \left(\frac{r^{2}+a^{2}}{b}\right)+4\left(\tilde{v}_{0}^{2}-1\right) r^{2}-4 a^{2}} \\
\tilde{h}^{2} & =\frac{r^{4}\left[\tilde{v}_{0}^{2} \ln \left(\frac{r^{2}+a^{2}}{b}\right)-4\right]^{4}\left[\tilde{v}_{0}^{2} \ln \left(\frac{r^{2}+a^{2}}{b}\right)+6 \tilde{v}_{0}^{2}-4\right]}{128\left[\left(a^{2}-r^{2}\right) \tilde{v}_{0}^{2} \ln \left(\frac{r^{2}+a^{2}}{b}\right)+4 r^{2}\left(1-2 \tilde{v}_{0}^{2}\right)-4 a^{2}\right]}
\end{aligned}
$$

where $\tilde{\rho}=\frac{4 \pi G}{c^{2}} \rho, \tilde{p}=\frac{8 \pi G}{c^{4}} p, \tilde{v}_{c}^{2}=v_{c}^{2} / c^{2}, \tilde{v}_{0}^{2}=v_{0}^{2} / c^{2}$ and $\tilde{h}^{2}=h^{2} / c^{2}$. In order for the weak energy condition $\rho \geq 0$ to be satisfied a cut-off radius $r_{c}$ must be imposed such that $r_{c}^{2}+a^{2}=b$. Again, the other relationships will be analyzed using a graphic method. In figure $4(a)$ we graph, as functions of $r$, the relativistic energy density $\tilde{\rho}$, the Newtonian 
density profile $\tilde{\rho}_{N}$ and the isotropic pressure $\tilde{p}$ for dark matter haloes constructed from a logarithmic seed potential with parameters $\tilde{v}_{0}^{2}=0.458, a=3$ and $r_{c}=10$. We observe that the energy density presents a maximum at the center of the distribution of matter, and then decreases rapidly with the radial distance $r$ which permits us to define a cut-off radius $r_{c}$ and so, in principle, consider the structure as a compact object. We also see that the relativistic effects decrease the density profile everywhere of the dark halo and they become more important in the central region of the distribution. At large $r$ both density profiles (relativistic and Newtonian) coincide. For these values of the parameters also we have positive stresses (pressure).

In figure $4(b)$ we show the relativistic circular speed $\tilde{v}_{c}^{2}$, the Newtonian rotation curve $\tilde{v}_{c N}^{2}=v_{c N}^{2} / c^{2}$ and the specific angular momentum $\tilde{h}^{2} \times 10^{-4}$ for the same values of parameters, also as function of $r$. In contrast to the energy density, we find that the relativistic effects increase everywhere the circular speed of the particles and they become more important as we move away from the central region. We also see that relativistic rotation curve is flattened after a certain value of $r$ as observational data indicate. The speed of the particles always is less than light speed (dominant energy condition). We find that for these values of parameters the motion of particles is stable against radial perturbations.

\section{CONCLUSIONS}

Perfect fluid sources for static spherically symmetric fields in isotropic coordinates from given solutions of Poisson's equation were investigated. The method was illustrated with three simple examples based on the potential-density pairs corresponding to a harmonic oscillator, the well-known Plummer model and a massive spherical dark matter halo model with a logarithmic potential.

The geodesic circular motion of test particles around such structures was also studied. We found that the relativistic effects increase everywhere the speed of particles, and are more significant as we move away from the central region in the case of the first and third model, in the regions close to its maximum value in the case of Plummer type fields and, as expected, for velocities comparable to the speed of light. For logarithmic potential type spheres we also found that relativistic rotation curve is flattened after a certain value of the radial distance as observational data indicate.

Moreover, the stability of the orbits against radial perturbations was analyzed using an extension of the Rayleigh criteria of stability of a fluid at rest in a gravitational field. In all the cases we found stable circular orbits, but for harmonic oscillator and Plummer type fields was observed that the increase in the gravitational field can make unstable the motion of the particles. The models considered satisfy all the energy conditions.

\section{References}

[1] M. I. Wilkinson, J. Kleyna, N. W. Evans and G. Gilmore, MNRAS 330, 778 (2002).

[2] A. T. Bajkova and V. V. Bobylev, Astronomy Letters 42, No. 9, 567 (2016).

[3] V. V. Bobylev, A. T. Bajkova and A. O. Gromov, Astronomy Letters 43, No 4, pp. 241 (2017).

[4] W. Dehnen, MNRAS 265, 250 (1993).

[5] S. Tremaine et al., Astron. J. 107, 634 (1994).

[6] H. C. Plummer, MNRAS 71, 460 (1911).

[7] L. Hernquist, Ap. J., 356, 359 (1990).

[8] J. F. Navarro, C. S. Frenk and S. D. M. White, Ap. J. 462, 563 (1996).

[9] M. S. R. Delgaty and K. Lake, Comput. Phys. Commun. 115, 395 (1998).

[10] H. Stephani, D. Kramer, M. McCallum, C. Hoenselaers, and E. Herlt, Exact Solutions of Einsteins's Field Equations (Cambridge University Press, Cambridge, England, 2003).

[11] D. Vogt and P. S. Letelier, Mon. Not. Roy. Astron. Soc. 402, 1313 (2010).

[12] D. Vogt and P. S. Letelier, Mon. Not. Roy. Astron. Soc. 406, 2689 (2010).

[13] P. H. Nguyen, M. Lingam, Mon. Not. Roy. Astron. Soc. 436, 3, 2014 (2013).

[14] G. García-Reyes, Gen. Relativ. Gravit. 49, 3, 1-13 (2017).

[15] G. García-Reyes and Kevin A. Hernández-Gómez, Int. J. Mod. Phys. D, Vol. 27, Issue 07, 1850068-1 (2018).

[16] R. C. Tolman, Relativity, Thermodynamics, and Cosmology (Oxford University Press, London, 1934).

[17] Ya. B. Zeldovich and J. D. Novikov, Relativistic Astrophysics, Vol. I: Stars and Relativity (University of Chicago Press, Chicago, 1971).

[18] T. Matos, D. Núñez and R. A. Sussman, Class. Quant. Grav. 21, 22, 5275 (2004).

[19] L. D. Landau and E. M. Lifshitz, Classical Theory of Fields (Pergamon, Oxford, 1976). 


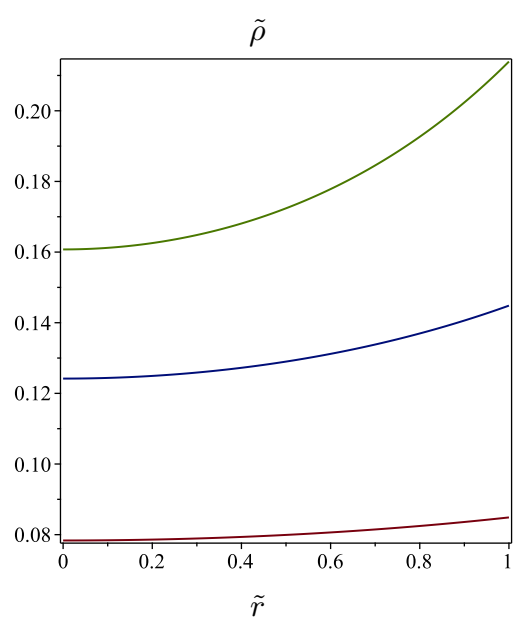

(a)

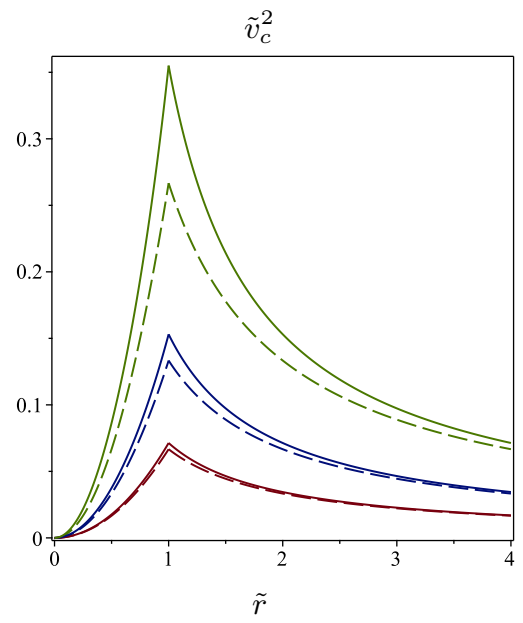

(c)

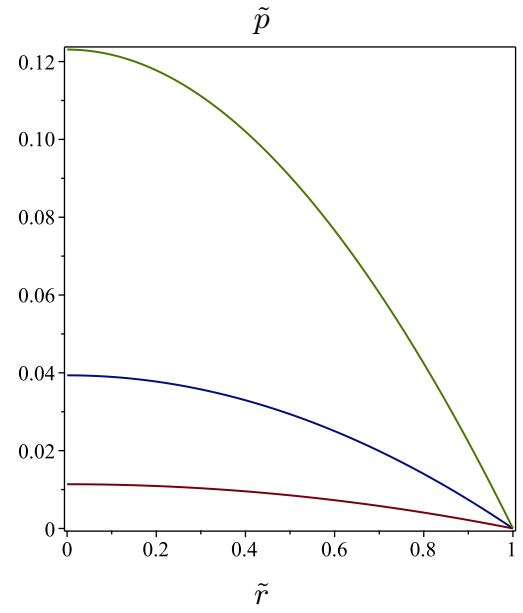

(b)

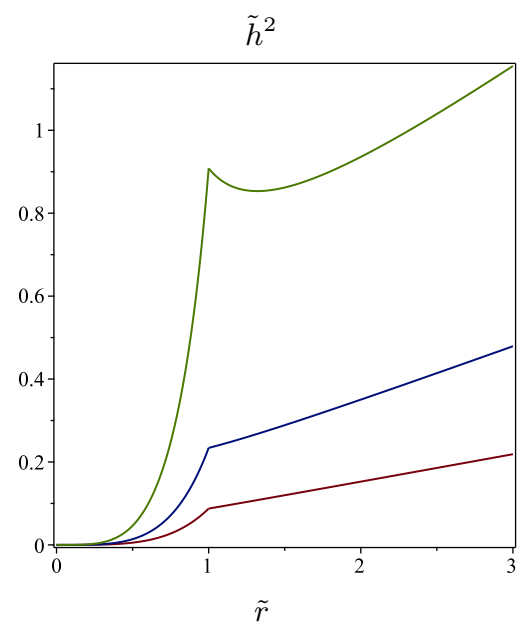

$(d)$

FIG. 1: For the relativistic analogue of a homogeneous sphere we plot, as functions of $\tilde{r},(a)$ the energy density $\tilde{\rho},(b)$ the isotropic pressure $\tilde{p}$ with parameter $b=0.1$ (bottom curves), $0.2,0.4$ (top curves), (c) the relativistic circular speed $\tilde{v}_{c}^{2}$ (solid curves), the Newtonian rotation curves $\tilde{v}_{c N}^{2}$ (dashed curves) with $b=0.1$ (bottom curves), $0.2,0.4$ (top curves), and ( $d$ ) the specific angular momentum $\tilde{h}^{2}$ for the same value of the parameter $b=0.1$ (bottom curve), $0.2,0.4$ (top curve).

[20] L. D. Landau and E. M. Lifshitz, Fluid Mechanics (Addison-Wesley, Reading, MA, 1989).

[21] A. H. Taub, Ann. Rev. Fluid Mech. 10, 301 (1978).

[22] J. L. Synge, Relativity: The General Theory (NorthHolland, Amsterdam, 1966).

[23] C. W. Misner, K. S. Thorne and J. A. Wheeler, Gravitation (Freeman, San Francisco, 1973).

[24] S. W. Hawking and G. F. R. Ellis, The large-scale structure of space-time (Cambridge University Press, Cambridge, 1973).

[25] B. Schutz, A First Course in General Relativity (Cambridge University Press, Second Edition, 2009).

[26] P. Kustaanheimo and B. Qvist, Gen. Relativ. Gravit. 30, No. 4, 663 (1998).

[27] Lord Rayleigh, 1917, Proc. R. Soc. London A, 93, 148.

[28] P. S. Letelier, Phys. Rev. D 68, 104002 (2003).

[29] G. Darmois, Mémorial des Sciences Mathématiques (Gauthier-Villars, Paris, 1927) Fasc. 25.

[30] W. B. Bonnor and P. A. Vickers, Gen. Relativ. Gravit 13 (1), 291981.

[31] M. Miyamoto and R. Nagai, Publ. Astron. Soc. Jpn. 27, 533 (1975).

[32] R. Nagai and M. Miyamoto, Publ. Astron. Soc. Jpn. 28, 1 (1976).

[33] H. A. Buchdahl, Astrophys. J. 140, 1512 (1964).

[34] J. Binney, Mon. Not. R. Astron. Soc. 196, 455 (1981). 


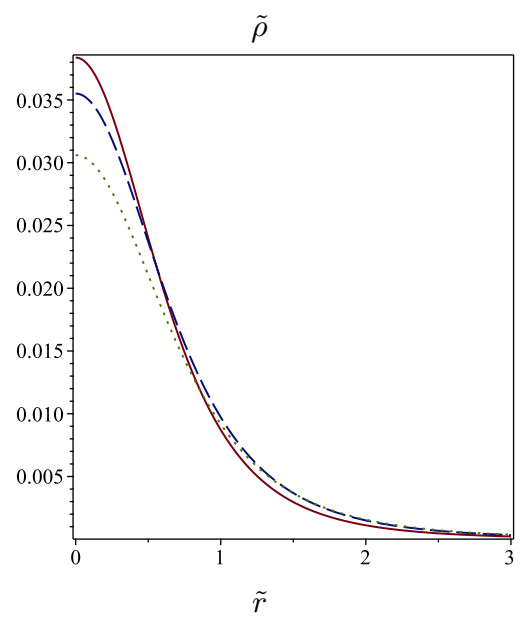

(a)

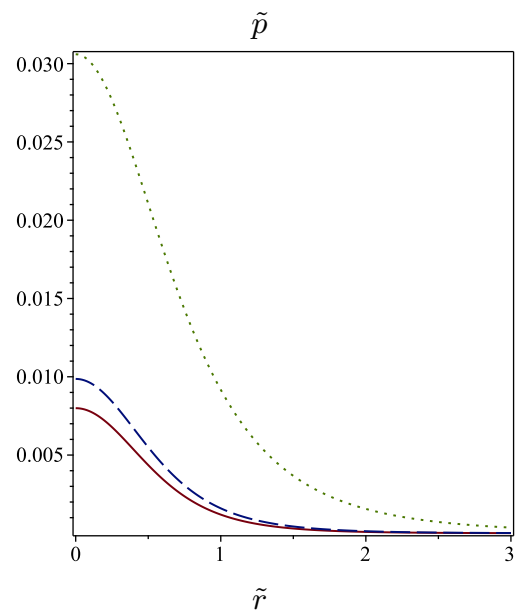

$(c)$

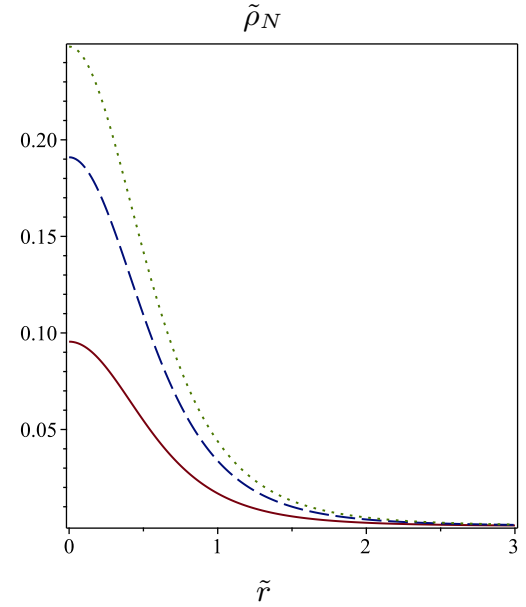

(b)

FIG. 2: The relativistic and Newtonian density profiles $(a) \tilde{\rho},(b) \tilde{\rho}_{N}$ and $(c)$ the isotropic pressure $\tilde{p}$ for Plummer type spheres with parameter $b=0.2$ (solid curves), 0.4 (dashed curves), 0.52 (dotted curves), as functions of $\tilde{r}$. 


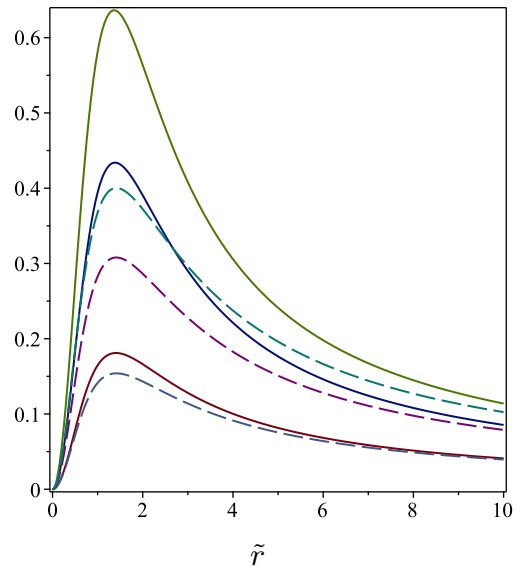

(a)

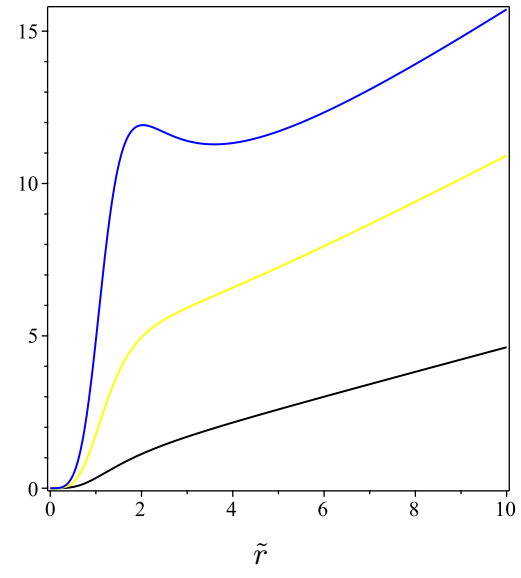

(b)

FIG. 3: (a) The relativistic and Newtonian circular speed $\tilde{v}_{c}^{2}$ (solid curves) and $\tilde{v}_{c N}^{2}$ (dashed curves), and (b) the specific angular momentum $\tilde{h}^{2}$ for Plummer type spheres with parameters $b=0.2$ (bottom curves), $0.4,0.52$ (top curves), as functions of $\tilde{r}$.

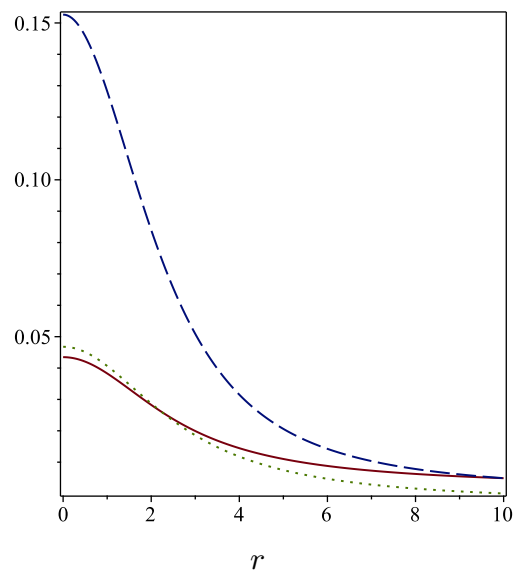

$(a)$

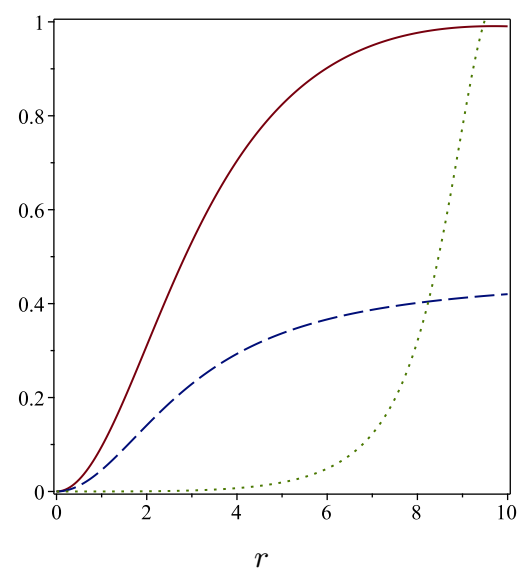

(b)

FIG. 4: (a) The relativistic energy density $\tilde{\rho}$ (solid curve), Newtonian density distribution $\tilde{\rho}_{N}$ (dashed curve), the isotropic pressure $\tilde{p}$ (dotted curve), (b) the relativistic circular speed $\tilde{v}_{c}^{2}$ (solid curve), Newtonian rotation curve $\tilde{v}_{c N}^{2}$ (dashed curve) and the specific angular momentum $\tilde{h}^{2} \times 10^{-4}$ (dotted curve), for logarithmic potential type dark matter haloes with parameters $\tilde{v}_{0}^{2}=0.458, a=3$ and $r_{c}=10$, as functions of $r$. 